\title{
Beta limits in long-pulse tokamak discharges*
}

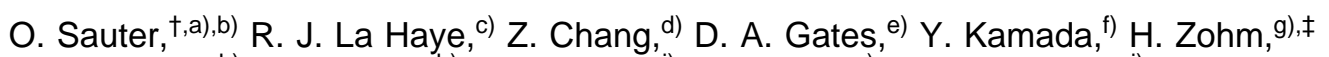

A. Bondeson, ${ }^{\text {h) }}$ D. Boucher, ${ }^{\text {b) }}$ J. D. Callen, ${ }^{\text {, }}$ M. S. Chu, ${ }^{\text {c) }}$ T. A. Gianakon, ${ }^{\text {i) }}$ O. Gruber, ${ }^{\text {g) }}$

R. W. Harvey, ${ }^{\text {) }}$ C. C. Hegna, ${ }^{\text {i) }}$ L. L. Lao, ${ }^{\text {c) }}$ D. A. Monticello, ${ }^{\text {d) }}$ F. Perkins, ${ }^{\text {b) }}$

A. Pletzer, ${ }^{\text {a) }}$ A. H. Reiman, ${ }^{\text {d) }}$ M. Rosenbluth, ${ }^{\text {b) }}$ E. J. Strait, ${ }^{\text {c) }}$ T. S. Taylor, ${ }^{\text {c) }}$

A. D. Turnbull, ${ }^{c)}$ F. Waelbroeck, ${ }^{k}{ }^{k}$ J. C. Wesley, $\left.{ }^{b}\right)$ H. R. Wilson, ${ }^{e)}$ and R. Yoshinof)

${ }^{a)}$ CRPP, Assoc. Euratom-Switzerland, PPB-Ecublens, 1015 Lausanne, Switzerland

${ }^{b)}$ ITER-JCT, 11025 N Torrey Pines Rd., La Jolla, California 92037

${ }^{c)}$ General Atomics, P.O. Box 85608, San Diego, California 92186-5608

${ }^{d)}$ Princeton Plasma Physics Laboratory, Princeton, New Jersey 08540

${ }^{e)}$ UKAEA Fusion, Culham, Abingdon, Oxfordshire, OX14 3DB, United Kingdom

f) JAERI, Naka-machi, Naka-gun, Ibaraki-ken, 311-01, Japan

${ }^{g}$ MIP für Plasmaphysik, EURATOM Association, 85748 Garching, Germany

${ }^{h)}$ Chalmers University of Technology, Göteborg, Sweden

${ }^{i)}$ University of Wisconsin, Madison, Wisconsin 53706-16787

${ }^{j)}$ CompX, 12839 Via Grimaldi, Del Mar, California 92014

${ }^{k)}$ IFS, University of Texas, Austin, Texas 78712

(Received 12 November 1996; accepted 23 January 1997)

The maximum normalized beta achieved in long-pulse tokamak discharges at low collisionality falls significantly below both that observed in short pulse discharges and that predicted by the ideal MHD theory. Recent long-pulse experiments, in particular those simulating the International Thermonuclear Experimental Reactor (ITER) [M. Rosenbluth et al., Plasma Physics and Controlled Nuclear Fusion (International Atomic Energy Agency, Vienna, 1995), Vol. 2, p. 517] scenarios with low collisionality $\nu_{e^{*}}$, are often limited by low- $m / n$ nonideal magnetohydrodynamic (MHD) modes. The effect of saturated MHD modes is a reduction of the confinement time by $10 \%-20 \%$, depending on the island size and location, and can lead to a disruption. Recent theories on neoclassical destabilization of tearing modes, including the effects of a perturbed helical bootstrap current, are successful in explaining the qualitative behavior of the resistive modes and recent results are consistent with the size of the saturated islands. Also, a strong correlation is observed between the onset of these low- $m / n$ modes with sawteeth, edge localized modes (ELM), or fishbone events, consistent with the seed island required by the theory. We will focus on a quantitative comparison between both the conventional resistive and neoclassical theories, and the experimental results of several machines, which have all observed these low- $m / n$ nonideal modes. This enables us to single out the key issues in projecting the long-pulse beta limits of ITER-size tokamaks and also to discuss possible plasma control methods that can increase the soft $\beta$ limit, decrease the seed perturbations, and/or diminish the effects on confinement. () 1997 American Institute of Physics. [S1070-664X(97)94005-2]

\section{INTRODUCTION}

The ideal magnetohydrodynamic (MHD) theory has been shown to adequately predict the upper bound for the beta limit in present day tokamak discharges, without wall stabilization and neglecting the $m=1 / n=1$ internal mode related to sawteeth activity and stabilized by nonideal effects. ${ }^{1}$ The ideal limit can be expressed, for monotonic $q$ profiles, as $\beta_{N} \equiv \beta(\%) /[I(\mathrm{MA}) / a(\mathrm{~m}) B(\mathrm{~T})] \approx 4 l_{i}$, where $l_{i}$ is the internal plasma inductance. This limit has been reached in most of the tokamaks and can be increased with the help of sufficient toroidal rotation and a nearby conducting wall. ${ }^{2}$ However, these discharges have only been transient. In more recent experiments, when trying to maintain a high beta discharge for many confinement times, low- $m / n$ resistive modes have limited the achievable beta. ${ }^{3-8}$ A typical scenario is shown in Fig. 1. At a constant density, the power is

\footnotetext{
*Paper 6IB3, Bull. Am. Phys. Soc. 41, 1528 (1996).

Invited speaker. Presently assigned to ITER-JCT San Diego under a VHTP agreement.

†Permanent address: IPF, Universitaet Stuttgart, Pfaffenwaldring 31, D-70569 Stuttgart, Germany.
}

stepped up and, typically after a sawtooth crash or an ELM, a low- $m / n$ mode is excited. In this case a $3 / 2$, mode is onset at $t=2280 \mathrm{~ms}$ and grows on a resistive time scale to a saturated island width $\left(w_{\text {sat }}\right)$, inducing a drop in the stored energy such as $\Delta \tau_{e} / \tau_{e} \equiv-4\left(\rho_{s} / a\right)^{4} w_{\text {sat }} / \rho_{s}$, where $\tau_{e}$ is the energy confinement time, $\rho_{s}$ is the radius of the $q=m / n$ flux surface, and $a$ the minor plasma radius. ${ }^{9}$ This will be referred to as a soft beta limit. In this shot the injected power is further increased and the $3 / 2$ mode increases, but more importantly a $2 / 1$ mode is destabilized. This mode grows to a large size such that the rotation slows down and eventually locks, inducing a disruption. This is referred to as a hard beta limit.

The typical value of $\beta_{N}$, at which these resistive modes appear is in the range 1.5-2.5 in discharges similar to the International Thermonuclear Experimental Reactor (ITER) scenarios, high confinement modes ( $\mathrm{H}$ modes) with edge localized modes (ELM), and at a collisionality, $\nu_{e} \approx n_{e} q R /$ $\epsilon^{3 / 2} T_{e}^{2}$, similar to the one expected for ITER reference scenarios. The ideal $\beta_{N}$ limit for ITER scenarios is $3.5-4,{ }^{10}$ while it requires only $\beta_{N} \geqslant 2$ to be able to ignite. However, this value is exactly in the range where the resistive modes 


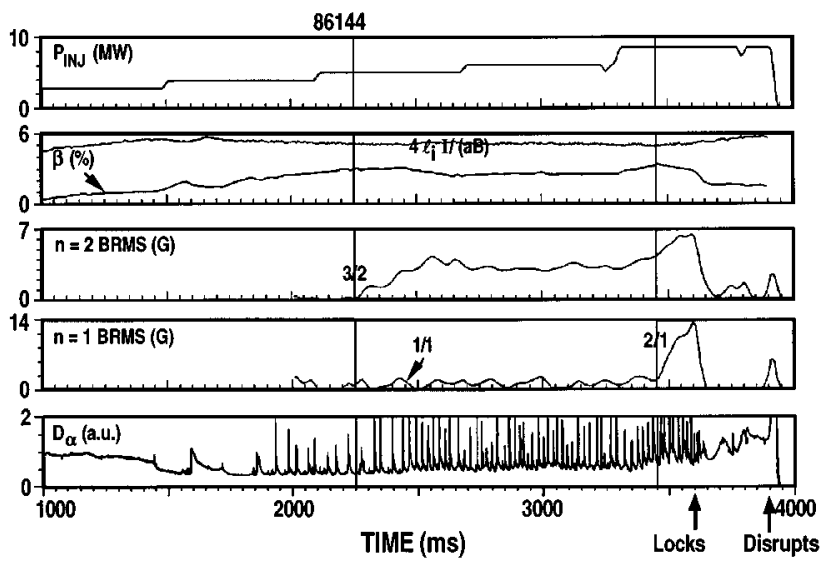

FIG. 1. Typical time history of the neoclassical modes. In this DIII-D discharge \#86144, a $3 / 2$ mode is excited at $t=2250 \mathrm{~ms}$ and induces a saturation of $\beta$. At $t=3450 \mathrm{~ms}$, a $2 / 1$ mode grows to a large amplitude until it locks and triggers a disruption. The computed ideal limit for this case is $\beta_{N}$ $\approx 3.4$.

can appear and limit the operational $\beta_{N}$ below 2 . This is why it is important for projecting ITER performance to be able to predict the physics of these modes. The neoclassical tearing mode theory was first developed ten years ago,,${ }^{11,12}$ based on neoclassical MHD, ${ }^{13(a)}$ and was reemphasized recently, ${ }^{13(b)}$ showing the destabilizing effect of the perturbed bootstrap current, but was correlated to the evolution of the saturated island width of resistive modes in the experiment only a few years ago in the Tokamak Fusion Test Reactor (TFTR). ${ }^{6}$ They have also been identified as a soft and/or hard beta limiting process in Axisymmetric Diverter ExperimentUpgrade (ASDEX-U), ${ }^{5}$ COMPASS-D,${ }^{8}$ DIII-D,${ }^{7}$ and JT60-U (Japan Torus-60 Upgrade). ${ }^{4}$ This has stimulated much theoretical work as well, ${ }^{13-19}$ related in particular to the threshold island size of the seed island required by the theory, including the effect of finite ratio of perpendicular to parallel transport, $\chi_{\perp} / \chi_{\|},{ }^{14,15}$ and drift and polarization current effects. ${ }^{16-19}$

Neoclassical tearing modes are not the only modes capable of limiting the operational beta. Other MHD effects can occur even at relatively low beta, such as frequent giant ELMs, $\beta_{p}$ collapse, or external kink modes. ${ }^{4,20,21}$ These are, in general, related to the ideal MHD limit, which can be lower than $\left(4 l_{i}\right)$ if the profiles are not optimized. However, the ITER reference scenarios are clearly ideally stable with respect to external kink modes and to ballooning modes in the core region. The axis safety factor is below unity, $q_{0}<1$ and the pressure gradient near the edge is limited by the ballooning criteria, thus sawteeth and ELMs are expected in ITER, which can trigger finite-size seed islands at the $q=m / n$ surfaces. Therefore the conventional resistive and neoclassical modes are the most likely candidate to limit the operational beta in ITER. We shall therefore concentrate on this topic in this paper. We aim to determine whether the ITER design range, $\beta_{N}=2-2.5$ that is $30 \%-40 \%$ below the ideal limit, is reasonable and can be sustained in such longpulse monotonic $q$ discharges (1000 s). Therefore we have analyzed the different aspects of the theory in a comprehensive way and compared the predictions with the results from
ASDEX-U, COMPASS-D, DIII-D, JT60-U, and TFTR. This provides us with a wide range of plasma parameters that permits identification of those parts of the theory that can be used with confidence to extrapolate to ITER and isolates the specific uncertainties limiting the accuracy of the predictions.

The physics of the neoclassical island evolution equation and the comparison with experiments is described in Sec. II, where we show that the driving term explains the island evolution very well and show how to distinguish these modes from the conventional resistive theory. We discuss the projection to a plasma of the size and characteristics predicted for ITER in Sec. III, where we see that the predicted seed island threshold is $2-6 \mathrm{~cm}$ and therefore that neoclassical modes can be expected to be unstable in ITER. However, we show that the growth time is of the order of $100 \mathrm{~s}$, which gives plenty of operational time for profile or feedback control. We also present a preliminary study, indicating that local electron cyclotron current drive (ECCD) can be used to stabilize the modes. Finally, in Sec. IV, we summarize the results and discuss theoretical and experimental work needed to gain confidence in the predictions to ITER.

\section{NEOCLASSICAL TEARING THEORY AND EXPERIMENTAL OBSERVATIONS}

\section{A. Modified Rutherford equation}

The nonlinear island evolution in the "conventional" resistive theory is given by the Rutherford equation: ${ }^{22,23}$

$$
\begin{aligned}
\frac{\tau_{R}}{\rho_{s}} \frac{d w}{d t} & =\rho_{s} \Delta^{\prime}(w)-\beta_{p} \rho_{s} \frac{a_{\mathrm{GGJ}}}{w} \\
& \equiv \rho_{s}\left(\Delta^{\prime}-\alpha w\right)-\beta_{p} \rho_{s} \frac{a_{\mathrm{GGJ}}}{w},
\end{aligned}
$$

where $\tau_{R}=\mu_{0} \rho_{s}^{2} / 1.22 \eta_{\text {neo }} ; \eta_{\text {neo }}$ is the neoclassical resistivity; $\rho_{s}$ is the location of the $q=m / n$ flux surface; and $\rho$ is the radial coordinate related to the square root of the toroidal flux, $\rho=a\left(\Phi / \Phi_{a}\right)^{1 / 2} ; \quad \beta_{p}$ the local poloidal beta with $\beta_{p}=2 \mu_{0} p / B_{\mathrm{pol}}^{2}, B_{\mathrm{pol}}=(d \psi / d r) / R_{0}$, and $\psi$ the poloidal flux; $\Delta^{\prime}$ the usual tearing parameter, given by the jump in the logarithmic derivative of the radial magnetic field and driven mainly by the equilibrium current gradient; $\alpha$ is the reduction of this jump when the island is large enough to modify the equilibrium current profile; and the last term is the stabilizing Glasser-Green-Johnson (GGJ) effect due to the equilibrium pressure gradients and favorable curvature in the outer part of the island. ${ }^{24-26}$ Neoclassical effects such as the bootstrap current have to be included in Ohm's law, not only in the equilibrium calculation, but also in the stability calculation. ${ }^{11,12}$ Then a new driving term appears in Eq. (1) due to the flattening of the pressure profile inside the island and the reduction of the bootstrap current that follows. This results in a negative perturbed helical bootstrap current inside the island, which is destabilizing, as is sketched in Fig. 2: the equilibrium magnetic field near the rational surface $q=q_{s}=m / n$ is written in the vicinity of the resonant surface as $\mathbf{B} \cong \mathbf{B}_{s}+\mathbf{B}^{*}$, where 


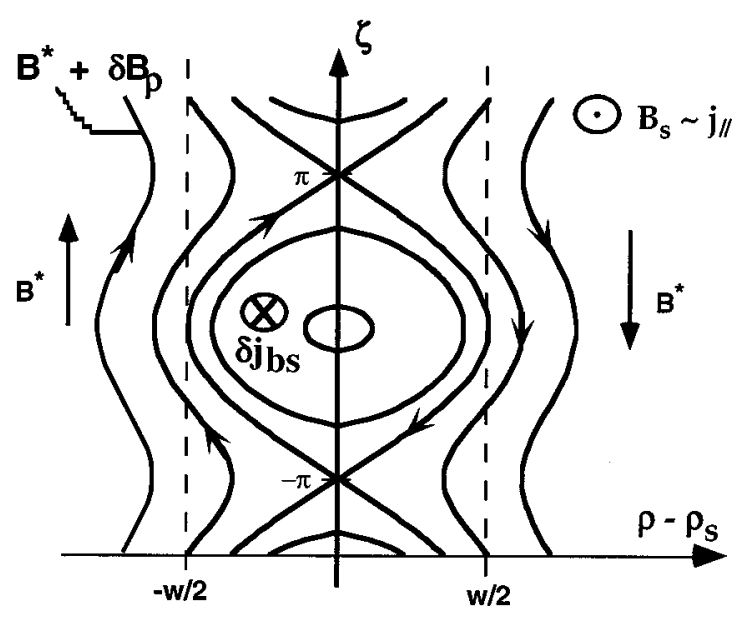

FIG. 2. Sketch of the helical component, $\zeta=\theta-n \varphi / m$, of the equilibrium magnetic field $\mathbf{B}^{*}$ near the resonant surface $\rho_{s}$, perturbed by a small radial field $\delta \mathbf{B}_{\rho}$. The equilibrium current is in the direction of $\mathbf{B}_{s}$, whereas the perturbed bootstrap current $\delta \mathbf{j}_{\mathrm{bs}}$ inside the island is in the opposite direction.

$$
B^{*}=B_{\theta}\left(1-\frac{q}{q_{s}}\right) \approx-\frac{q_{s}^{\prime}}{q_{s}}\left(\rho-\rho_{s}\right) B_{\theta}
$$

is the component in the helical direction $\zeta=\theta-n \varphi / m$ perpendicular to the magnetic field $\mathbf{B}_{s}$ at the rational surface, and the ' denotes the radial derivative with respect to $\rho$. If $\mathbf{B}^{*}$, shown with the straight arrows in Fig. 2 is perturbed with a small radial magnetic field $\delta B_{\rho}(\rho) \sin \zeta$, islands are formed and the new topology is shown as well. Due to the large parallel thermal conductivity, the temperature equilibrates on the new flux surfaces inside the island and thus flattens the radial profile. Therefore the bootstrap current, proportional to the radial temperature and density gradients, decreases and gives rise to a negative perturbed bootstrap current $\delta j_{\text {bs }}$, which has the same helical structure as the island. As seen in Fig. 2, $\delta j_{\text {bs }}\left(\sim-\mathbf{B}_{s}\right)$ reinforces the perturbed helical magnetic field and is therefore destabilizing. We note also from this figure that if $q^{\prime}$ is negative, then the arrows on the magnetic surfaces change direction, while $\delta j_{\text {bs }}$ remains unchanged, and the perturbed bootstrap current is stabilizing. ${ }^{11,12}$ If the island is small enough so that the perpendicular transport can compete with the transport along the magnetic field, then the pressure is less flattened and it reduces the driving term. Therefore there is a threshold island width, related to $\chi_{\perp} / \chi_{\|}$, below which the mode is stable, assuming $\Delta^{\prime}<0 .{ }^{14}$ The effect of the bootstrap current on the stability, and the dependence of the threshold island width on $\chi_{\perp} / \chi_{\|}$, has been confirmed in a three dimensions (3D) nonlinear noncircular toroidal code as well, ${ }^{27}$ where the neoclassical stress tensor has been included in the high beta reduced MHD equations used in the FARGO code. ${ }^{28}$

Several authors have also shown that inertia effects giving rise to a finite polarization current through the perturbed electrostatic potential are also important. ${ }^{16-18}$ They found that this term is large and stabilizing, assuming the island rotation $\omega \approx \omega_{e^{*}}$ in the frame rotating with the plasma fluid, where $\omega_{e^{*}}=T /\left(e B r_{s} L_{T e}\right)$ is the electron drift frequency and $L_{T e}=T_{e} / T_{e}^{\prime}$. However, a recent work indicates that this term is destabilizing and of smaller amplitude without interaction with the wall. ${ }^{19}$ This work has pointed out an inconsistency between the form of the velocity profile across the island separatrix and the other assumptions used in the model, and therefore more physics in the kinetic treatment is needed to resolve this issue. With this caution we include the polarization term and study its effect. Altogether the modified Rutherford equation is given by ${ }^{14,8,15}$

$$
\begin{aligned}
\frac{\tau_{R}}{\rho_{s}} \frac{d w}{d t}= & \rho_{s} \Delta^{\prime}(w)+\beta_{p} \rho_{s}\left(-\frac{\hat{a}_{\mathrm{GGJ}}(w)}{w}\right. \\
& +\sum_{A=n_{e}, T_{e}, T_{i}} a_{\mathrm{bs}, A}\left(\nu_{e^{*}}\right) \frac{w}{w^{2}+w_{d, A}^{2}\left(\nu_{e^{*}}\right)} \\
& \left.-\frac{\hat{a}_{\mathrm{pol}}(w, g)}{w^{3}}\right),
\end{aligned}
$$

with

$$
\begin{aligned}
& \hat{a}_{\mathrm{GGJ}}(w) \cong 6 \frac{D_{R}}{\beta_{p}} h_{1}(w) \approx 6 \frac{\epsilon^{2}}{s} \frac{L_{q}}{-L_{p}}\left(1-\frac{1}{q^{2}}\right) h_{1}(w), \\
& a_{\mathrm{bs}, A}\left(\nu_{e^{*}}\right) \approx a_{2}\left(-L_{\mathrm{bs}, A}\right) \frac{L_{q}}{-L_{p, A}}, \\
& w_{d} \approx 5.1 \rho_{s}\left(\frac{q}{\epsilon s m}\right)^{1 / 2}\left(\frac{\chi_{\perp}}{\chi_{\|}}\right)^{1 / 4}, \\
& \hat{a}_{\mathrm{pol}}(w, g) \approx a_{3}\left(\frac{L_{q}}{-L_{p}}\right)^{2} \rho_{p}^{2} g\left(\epsilon, \nu_{i}\right) h_{3}(w) ; \\
& g\left(\epsilon, \nu_{i}\right)=\left\{\begin{array}{cc}
1 \quad \text { if } \frac{\nu_{i}}{\epsilon \omega_{e^{*}}} \geqslant 0.3, \\
\epsilon^{3 / 2} \quad \text { otherwise, }
\end{array}\right.
\end{aligned}
$$

where $D_{R}=E+F$ is defined in Refs. 24-25; $\nu_{i}=8.16$ $\times 10^{7} n_{e 20} Z_{\text {eff }} / T_{i} /\left(T_{i} m_{i}\right)^{1 / 2}$ is the ion collision frequency; $L_{q}=q / q^{\prime}=\rho_{s} / s ; L_{p}=p / p^{\prime} ; \epsilon=a / R_{0} ; R_{0}$ is the major radius; the coefficients $a_{2}=3.2$ and $a_{3}=9.3$, from the large aspect ratio results, ${ }^{14,18}$ are used here as free parameters of order 1 to fit the experiment and to compensate the uncertainty on the value of $\Delta^{\prime}$; and the terms with a subscript $A$ are defined below. Note that $\Delta^{\prime}, D_{R}$ and the bootstrap current driving term $a_{\mathrm{bs}}$ can be computed using noncircular toroidal codes like PEST-III ${ }^{29}$ and CHEASE. ${ }^{30}$ Therefore one can expect a constant value of $a_{2}$ valid for all the different geometries and collisional regimes. We have taken $a_{2}=2.6$ and $a_{3}=5$, similar to Refs. 6 and 8, respectively. For $\rho_{s} \Delta^{\prime}$ we use the results from cylindrical or large- $m^{31}$ calculations when a reconstructed equilibrium is available and otherwise use $\rho_{s} \Delta^{\prime}=-m$, which is a typical value one obtains from the cylindrical calculation, at least for $m \geqslant 4$, while $(-2 m)$ would give a lower bound. The exact form of the GGJ term when the pressure profile is not flattened due to finite $\chi_{\perp} / \chi_{\|}$has still to be determined; that is why we have introduced a factor $h_{1}(w)=w^{2} /\left(w^{2}+w_{d \mathrm{GGJ}}^{2}\right)$ similar to the modification of the bootstrap current term. It would be interesting to extend the work done in Ref. 26 and determine whether $w_{d \mathrm{GGJ}}$ is smaller than $w_{d}$ or if $h_{1}$ has a different form. Note that even though $a_{\mathrm{GGJ}}$ is two to three times smaller than $a_{\text {bs }}$, it has to be included when comparing with experiment. 
Moreover, it has a different dependence on $\epsilon$ (due to its $\beta$ rather than $\beta_{p}$ dependence), which can enhance its importance at a large inverse aspect ratio. For $w_{d}$ we use $\chi_{\perp}=0.5^{*}\left(1+\kappa^{2}\right) * a^{2} / 6 \tau_{\text {thITER93 }},{ }^{32}$ and $\chi_{\|}=v_{T e}^{2} \tau_{e}$, except when mentioned otherwise. Different forms of $\chi_{\perp}$ and $\chi_{\|}$ have been used and they give about the same value for $w_{d}$ within a factor 2 .

As mentioned above, the exact form and sign of the polarization term is still under investigation, which is why we have introduced $h_{3}(w)$ to keep a general form. If $h_{3}=1$, one expects the value of $g$ to drop from 1 to $\epsilon^{3 / 2}$ at the onset of the mode when $\nu_{i} / \epsilon \omega_{e^{*}}<0.3,{ }^{18}$ while if the term has to be neglected we can set $g=0$. Therefore we shall use $h_{3}=1$ and $g=0$ or $\epsilon^{3 / 2}$ in the remainder of the paper. Note that the saturated width $w_{\text {sat }}$ does not depend on the exact form of $a_{\mathrm{GGJ}}$ and $a_{\mathrm{pol}}$ but only on their value at large $w$, and we obtain, assuming $\Delta^{\prime}<0$,

$$
w_{\mathrm{sat}}=\beta_{p} \frac{a_{\mathrm{bs}}-a_{\mathrm{GGJ}}}{-\rho_{s} \Delta^{\prime}} .
$$

This relation gives a good fit of the saturated island size of the $m / n$ resistive modes observed experimentally, ${ }^{6-8}$ and does not depend on the exact form of $\hat{a}_{\mathrm{GGJ}}$ and $\hat{a}_{\mathrm{pol}}$.

The terms $L_{\mathrm{bs}, A}$ come from the contribution of the density and temperature gradients in the bootstrap current. The total bootstrap current can be written as ${ }^{33}$

$$
\begin{aligned}
j_{\mathrm{bs} \|}= & \frac{p}{B_{p o}}\left(L_{31} \frac{n_{e}^{\prime}}{n_{e}}+R_{p e}\left(L_{31}+L_{32}\right) \frac{T_{e}^{\prime}}{T_{e}}+L_{31}\left(1+\alpha_{i}\right)\right. \\
& \left.\times\left(1-R_{p e}\right) \frac{T_{i}^{\prime}}{T_{i}}\right),
\end{aligned}
$$

where we have used $p_{i}=p-p_{e}, R_{p e}=p_{e} / p \approx 0.5$, and $L_{31}, L_{32}, \alpha_{i}$ depend on the effective trapped fraction and on $1 /\left(1+\nu_{e^{*}}^{1 / 2}+0.25 \nu_{e^{*}} / Z_{\mathrm{eff}}^{2}\right){ }^{34}$ Therefore $L_{\mathrm{bs}, A}$, for $A=n_{e}$, $T_{e}$, and $T_{i}$, is given by $L_{31}, R_{p e}\left(L_{31}+L_{32}\right)$, and $L_{31}\left(1+\alpha_{i}\right)\left(1-R_{p e}\right)$, respectively. Similarly, the term $1 / L_{p, A}$ in Eq. (4b) is given by $n_{e}^{\prime} / n_{e}, T_{e}^{\prime} / T_{e}$, and $T_{i}^{\prime} / T_{i}$. The corresponding value of $w_{d, A}$ is $w_{D n}$ and $w_{\chi i}$, as defined in Ref. 15 for $A=n_{e}$ and $T_{i}$, respectively, and $w_{d}$, Eq. (4c), for $A=T_{e}$. Here we shall consider only the flattening of $T e$ (The contribution from $n_{e}^{\prime}$ and $T_{i}^{\prime}$ are implicitly included in the free parameter $a_{2}$ and $n_{e}^{\prime}$ is very small in most experiments.) Therefore $L_{\mathrm{bs}}$ is then defined as $R_{p e}\left(L_{31}+L_{32}\right)$ and $A=T_{e}$ only. A database of high beta long-pulse discharges, ITERLPBL.DB1, with and without low- $m / n$ resistive modes is being assembled at the San Diego ITER-JCT working site (web page: http://picard.iterus.org/[mhd]). It contains at present 32 datasets from TFTR, 40 from DIII-D, 20 from COMPASS-D, 3 from ASDEX-U, and 2 from JT60-U. Using the effect of trapped particles and $Z_{\text {eff }}$, we obtain the following typical values for the coefficients in Eq. (5), using the local profile characteristics:

$$
L_{31} \approx-0.6 \pm 0.1 ; \quad L_{32} \approx 0.2 \pm 0.05 ; \quad \alpha_{i} \approx-0.65 \pm 0.1,
$$

which gives a typical value for $L_{\mathrm{bs}}$ of -0.2 , using $R_{p e}=0.5$. In comparison the expected values for ITER reference scenarios at $q=2$ are $L_{31}=-0.6, L_{32}=0.2$, and $\alpha_{i}=-0.55$, which are very similar, and, in particular, $L_{\mathrm{bs}}=-0.2$ as well. One notes that the density gradients are much more efficient in driving bootstrap current than temperature gradients and therefore ITER $\mathrm{H}$-mode reference scenarios, having flat density profiles, will have a smaller bootstrap fraction relative to some of the present experimental results. Note that ASDEX-U and DIII-D also have flat density profiles.

The collisionality enters in the definition of several parameters of Eq. (3) and is always such as to decrease the beta limit when decreasing $\nu_{e^{*}}$. This is why one can explain the $\nu_{e *}$ dependence of the observed beta limit, even with the different terms taken separately

(a) The width $w_{d}$ decreases with smaller $\nu_{e} *$ values and thus the island threshold decreases as well.

(b) The value of $g$ changes from a large value, $g=1$, in the collisional regime, to a small value, $g=\epsilon^{3 / 2}$, when $\nu_{i} / \epsilon \omega_{e} * 0.3$, and therefore dramatically reduces the critical island width as well.

(c) The bootstrap current increases at smaller $\nu_{e} *$ values, which is reflected in the $1 /\left(1+\nu_{e^{*}}^{1 / 2}\right)$ dependence of $L_{31}$ and $L_{32}$.

Let us now discuss in more detail Eq. (3) and its main characteristics. First, from the database, the typical range of values of the relevant coefficients are

$$
\begin{aligned}
& a_{\mathrm{GGJ}}=0.07-0.27, \\
& a_{\mathrm{bs}}=0.45-0.85, \\
& w_{d}=0.5-1.9(\mathrm{~cm}), \\
& a_{\mathrm{pol}}\left(g=\epsilon^{3 / 2}\right)=1-5\left(\mathrm{~cm}^{2}\right), \quad \text { except for TFTR, } \\
& \quad \text { which has } a_{\mathrm{pol}}=5-15\left(\mathrm{~cm}^{2}\right) .
\end{aligned}
$$

For COMPASS-D, we have used the "convective" form of $w_{d}$, since it is in a very collisionless regime. ${ }^{14}$ If we use the same form for the other tokamaks, $w_{d}$ is typically twice as large, and this gives the expected range of values of $w_{d}$. The value of $a_{\mathrm{pol}}$ is larger in TFTR due to more peaked profiles and a relatively larger value of the poloidal Larmor radius $\rho_{p}$ because of higher temperatures at $q=m / n$. These parameters for ITER are of the same order: $a_{\mathrm{GGJ}}=-0.25$, $a_{\mathrm{bs}}=1, w_{d}=2.5 \mathrm{~cm}$, and $a_{\mathrm{pol}}\left(g=\epsilon^{3 / 2}\right)=3$. Taking into account for the different possible expressions for $\chi_{\perp} / \chi_{\|}$, $w_{d} \approx 2-5 \mathrm{~cm}$ in ITER.

A typical plot of the right side of Eq. (3) is shown in Fig. 3 . Three curves are shown, using the typical parameters with $g=0$ [continuous line, Figs. 3(a) and 3(b)] and $g=\epsilon^{3 / 2}$ [dotted line, Fig. 3(b)], and at the critical $\beta_{p}$ value [dashed line Fig. 3(a)]. The value of $\beta_{p \text {, onset }}$ is taken from the TFTR shot 66873 at $t=3.90 \mathrm{~s}$, reproduced in Fig. 4 and that develops a $4 / 3$ mode of $5.2 \mathrm{~cm}$ saturated width. As mentioned above, $w_{\text {sat }}$ does not depend on $a_{\text {pol }}$ due to its $1 / w^{3}$ dependence, as seen in Fig. 3(b). The main effect of the polarization current is to change the island threshold width $w_{\text {crit }}$, and Fig. 3(b) illustrates the uncertainty in the theoretical predictions, typically in the range $0.2-3 \mathrm{~cm}$. The typical characteristic of these neoclassical tearing modes is a hysteresis behavior between the value of beta at the onset of the mode and the 

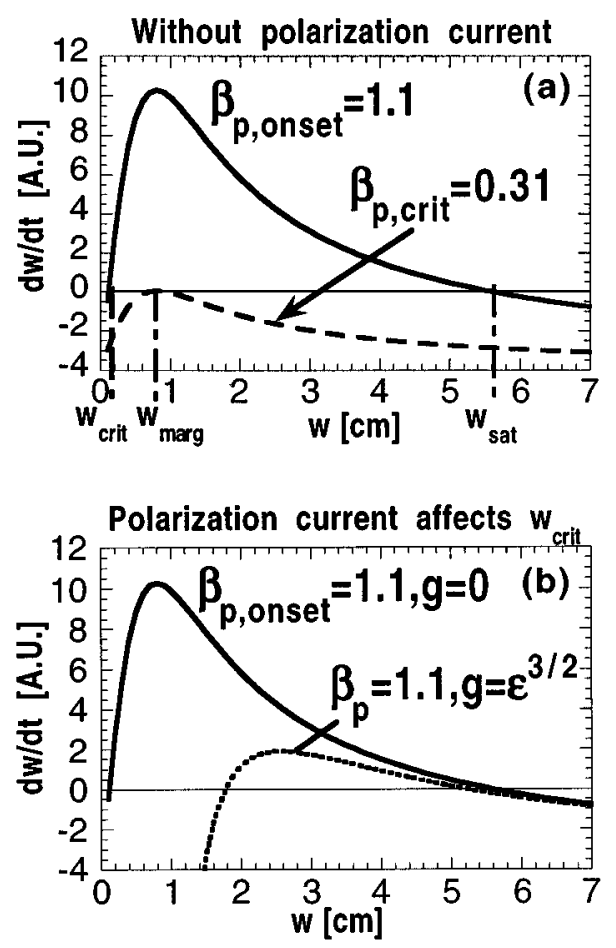

FIG. 3. The right-hand side of Eq. (3) with parameters similar to the TFTR shot 66873, $\rho_{s}=34 \mathrm{~cm}, \quad \rho_{s} \Delta^{\prime}=-4, \quad w_{d}=w_{d \mathrm{GGJ}}=0.8, \quad a_{\mathrm{GGJ}}=0.09$, $a_{\mathrm{bs}}=0.7$ : (a) $a_{\mathrm{pol}}=0$, and $\beta_{p}=1.1$ (continuous line) and $\beta_{p}=0.31$ (dashed line). The effect of $a_{\mathrm{pol}}$ is shown in (b), with $a_{\mathrm{pol}}=1, \beta_{p}=1.1$ (dotted line).

value at which the mode is stabilized, by lowering the input power while keeping the other plasma parameters constant. This can be understood from Fig. 3(a): At $\beta_{p}=\beta_{p \text {, onset }}$, a seed island of width $w_{\text {seed }}>w_{\text {crit }}$ is destabilized and grows to its saturated width $w_{\text {sat }}$. If the input power is then rampeddown, the island width decreases with $\beta_{p}$ and is stabilized at $\beta_{p}=\beta_{p, \text { crit }}<\beta_{p \text {, onset }}$. This hysteresis behavior has been seen in all the tokamaks mentioned earlier.

\section{B. "Conventional" and neoclassical tearing modes}

Let us now discuss how one can distinguish between a "conventional" resistive $m / n$ mode, having $\Delta^{\prime}>0$, and a

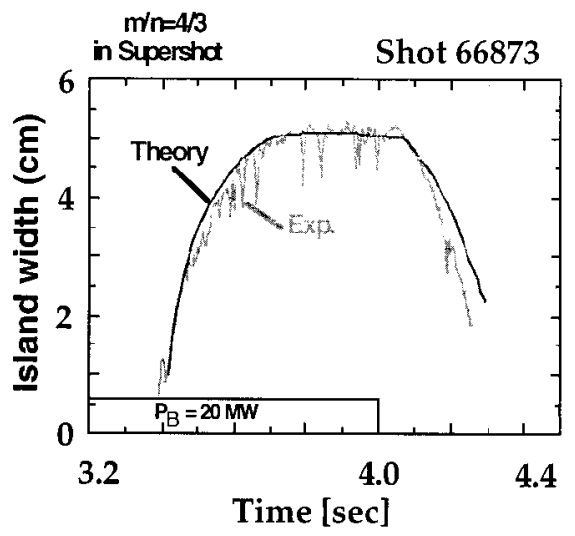

FIG. 4. Typical time evolution of a neoclassical mode $(m / n=4 / 3)$, including the decay when the power is turned off. This TFTR case was published in Ref. 6, Fig. 2(b). Note that $\beta_{p}$ decreases slightly between the onset and the saturated stage, due to the island. neoclassical tearing mode with $\Delta^{\prime}<0$, using the saturated island width and the growth time from the experiment. Naturally, one obvious way is to calculate $\Delta^{\prime}$ from an equilibrium reconstructed using experimental measurements and determine if it is positive or negative. However, $\Delta^{\prime}$ depends on the derivatives of the current or $q$ profile, and for marginal cases, it might be difficult to decide on the sign of $\Delta^{\prime}$. In this section, we quantify the accuracy on the value of $\Delta^{\prime}$ needed to distinguish between the two modes, using only the growing and saturated phase of the evolution. Dividing Eqs. (1) and (3) by $\left|\rho_{s} \Delta^{\prime}\right|$, normalizing the distance by $\rho_{s}$, $\bar{w}=w / \rho_{s}$, the time by $\tau_{\text {tear }}=\tau_{\mathrm{R}} /\left|\rho_{s} \Delta^{\prime}\right|, \bar{t}=t / \tau_{\text {tear }}$, and keeping only the terms necessary for recovering the saturated state, we can write the "conventional" Rutherford equation as:

$$
\frac{d \bar{w}}{d \bar{t}}=1-\frac{\bar{w}}{\bar{w}_{\text {satconv }}},
$$

with $\bar{w}_{\text {satconv }}=\Delta^{\prime} /\left(\alpha \rho_{s}\right)$, and the neoclassically modified Rutherford equation, as

$$
\frac{d \bar{w}}{d \bar{t}}=-1+\bar{w}_{\text {satneo }} \frac{\bar{w}}{\bar{w}^{2}+\bar{w}_{d}^{2}},
$$

with $\bar{w}_{\text {satneo }}=\beta_{\rho}\left(a_{\mathrm{bs}}-a_{\mathrm{GGJ}}\right) /\left|\rho_{s} \Delta^{\prime}\right|$. The island evolution due to each of these equations is shown in Figs. 5(a) and 5(b), respectively, for different values of $\bar{w}_{\text {satconv }}$ and $\bar{w}_{\text {satneo }}$, respectively. For Fig. 5(b), we chose $\bar{w}_{d}=0.025$, as in present tokamaks, and verified that using $\bar{w}_{d}=0.01$, as expected for ITER, does not change the curves significantly. Note that the mode does grow linear in $t$, in Fig. 5(a), and proportional to $t^{1 / 2}$ in Fig. 5(b), for small $t$, but due to the exponential saturation both type of curves cannot be distinguished experimentally for $w_{\text {sat }} / \rho_{s} \leqslant 0.2$. The typical values of $\tau_{\mathrm{R}}$ are $10-15 \mathrm{~s}$ for TFTR and JT60-U, $4 \mathrm{~s}$ for DIII-D, $2 \mathrm{~s}$ for ASDEX-U and $1 \mathrm{~s}$ for COMPASS-D, while $800 \mathrm{~s}$ for ITER.

In order to show how to use Fig. 5, we shall do it for the TFTR shot shown in Fig. 4. We have $w_{\text {sat }} / \rho_{s} \approx 0.15$ $\left(\rho_{s}=34 \mathrm{~cm}\right)$ and $80 \%$ of the saturated width is reached in about 0.2 s. From Fig. 5(a), one needs $\bar{t}=0.25$ to reach $80 \%$ of a $\bar{w}_{\text {sat }}=0.15$ island, that is a time $t=0.25 \tau_{\mathrm{R}} /\left|\rho_{s} \Delta^{\prime}\right|$. Therefore, with $\rho_{s} \Delta^{\prime} \approx 10$, one can model the island growth from the conventional resistive MHD. The same exercise from Fig. 5(b) gives $\bar{t} \simeq 0.15$, and thus one needs $\rho_{s} \Delta^{\prime} \approx-5$. Note that the latter value is approximately what was obtained using a cylindrical calculation in Ref. 6 $(-3.74)$ and is close to the typical value used here $(-m)$. One could argue that $\Delta^{\prime}$ should be computed using a twodimensional (2-D)-toroidal code like PEST-III, and Fig. 5, using the experimental values of $w_{\text {sat }} / \rho_{s}$ and of the growth time, gives the accuracy at which $\Delta^{\prime}$ has to be computed in order to distinguish between a "conventional" and a neoclassical tearing mode. Comparing also with some DIII-D shots, one typically needs $\left(\rho_{s} \Delta^{\prime}\right) \geqslant 2 m$ to model the mode growth with the conventional theory and $\rho_{s} \Delta^{\prime} \approx-m$ for the neoclassical case. Therefore $\Delta^{\prime}$ has to be evaluated with an accuracy better than $\pm m / \rho_{s}$. In the case of TFTR, due to its circular cross section and high- $m$ modes, preliminary comparison with PEST-III shows that the cylindrical $\Delta^{\prime}$ is 


\section{Conventional resistive MHD}

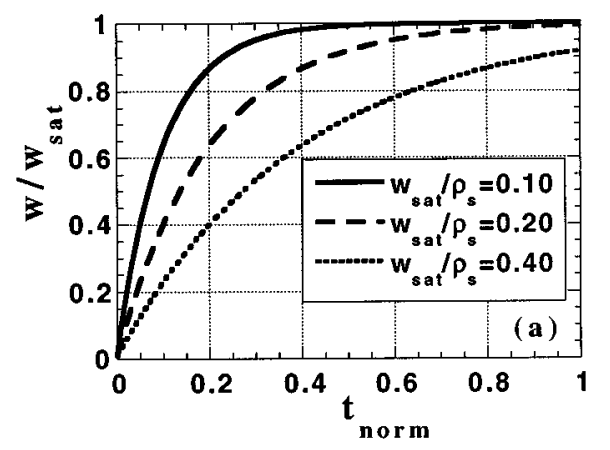

Neoclassical resistive MHD

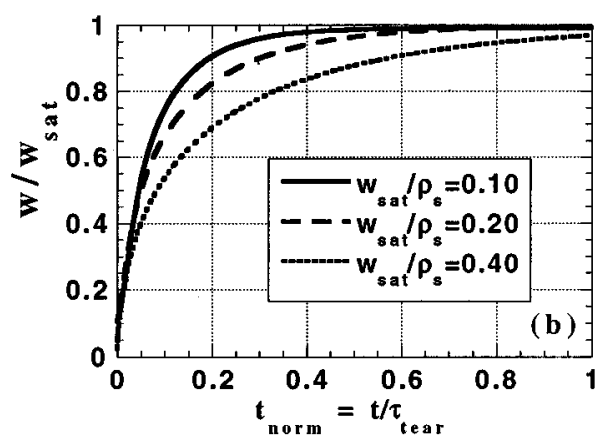

FIG. 5. Time evolution versus the normalized time $t, \bar{t}=t / \tau_{\text {tear }}$, with $\bar{\omega}_{\text {sat }}$ as parameters. No assumption on $\left|\rho_{s} \Delta^{\prime}\right|$, as it only enters in $\tau_{\text {tear }}$. (a) Evolution according to Eq. (9); (b) evolution according to Eq. (10) with $\bar{w}_{d}=0.025$. The faster growth in (b) is due to $t^{1 / 2}>t$ for $t<1$.

relevant. ${ }^{35}$ However, for the $2 / 1$ mode, and, in particular, in ITER-like geometry as in DIII-D, $\Delta^{\prime}$ is often found to be marginal, using PEST-III, and an accuracy of $\pm m / \rho_{s}$ is not easily obtained, due to the uncertainty on the reconstructed $q^{\prime}$ and $q^{\prime \prime}$, which can explain the discrepancy mentioned in Ref. 7.

However, another characteristic of the mode, which is its decay with decreasing $\beta_{p}$, allows one to distinguish between a "conventional" and a neoclassical tearing mode. This was shown in Ref. 6 for this TFTR shot 66873, where $\Delta^{\prime}$ and all the other parameters stayed constant, except for $\beta_{p}$ as the NBI power was turned off at $t=4 \mathrm{~s}$. Note that the change in beta is usually too small to affect the current and $q$ profiles, which is why $\Delta^{\prime}$ stays about constant. One should also mention that the GGJ term cannot explain the island evolution, since decreasing $\beta_{p}$ would reduce the stabilizing GGJ effect and thus would not reduce the island width. One sees in Fig. 4 that the neoclassical island evolution follows well both the growth and the decay of the mode, while keeping the free parameters fixed. As mentioned above, if $\beta_{p}$ is sufficiently decreased, below $\beta_{p \text {, crit }}$, then the mode should be stabilized. This is seen in all the mentioned experiments and an example from COMPASS-D is shown in Fig. $6{ }^{8}$ The ECRH power is ramped-down, $\beta_{p}$ decreases roughly linearly in time, and so does the island width. When $\beta_{p}<\beta_{p \text {, crit }}$, the mode is stabilized and its island width drops from $w_{\text {marg }}$, defined in Fig. 3, to zero. Thus $w_{\text {marg }}$ is the marginal saturated island width that can be sustained by the bootstrap

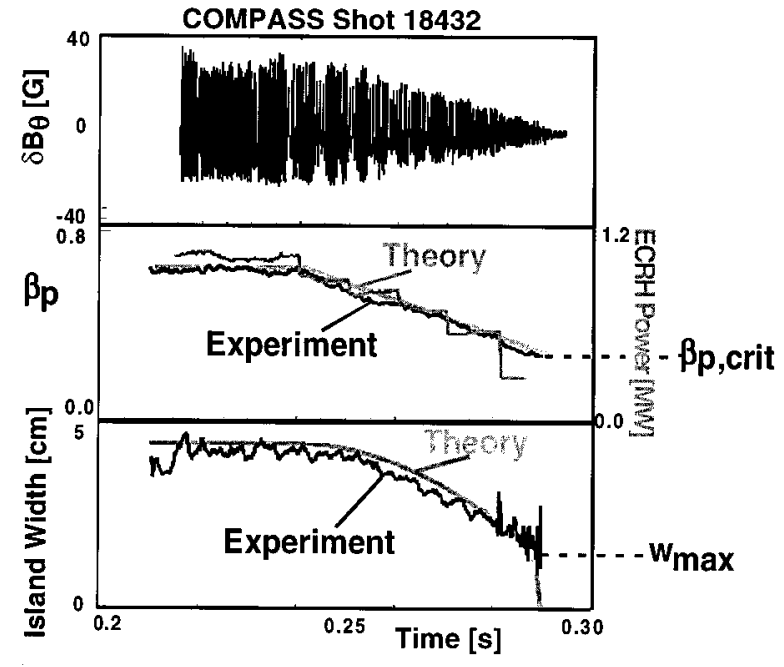

FIG. 6. Typical decay of the island size during a power ramped-down, shown for a COMPASS-D shot. At $t=0.29, \beta_{p}<\beta_{p \text {, crit }}$, the mode is stabilized and the island width drops very fast, consistent with the theoretical prediction, using the modified Rutherford equation (3). This figure was published in Ref. 8.

current driving term. If $\beta_{p}$ decreases or the collisionality increases, the island $w=w_{\text {marg }}$ decays away rapidly. Note that the "theory" curve can reproduce well the island decay and stabilization with or without the polarization term, as shown in Ref. 8, because the $\chi_{\perp} / \chi_{\|}$and polarization contributions are of the same order. Therefore $\beta_{p \text {,crit }}$ and $w_{\text {marg }}$ depend on the exact form at low $w$ of the coefficients in Eq. (4). However, a systematic study of $\beta_{p \text {, crit }}, w_{\text {marg }}$, and of the "drop-rate" of the island once $\beta_{p}<\beta_{p \text {,crit }}$ across different tokamaks should be sufficient to determine the relevance of the polarization term and the magnitude of the different terms. ${ }^{36}$ At present analysis, the polarization term is needed to explain COMPASS-D data, ${ }^{8}$ while it seems to give too large values of $w_{\text {marg }}$ in larger machines. However, the value of $a_{3}$ was determined in Ref. 8 using $w_{d}=0$, which increases $a_{3}$, and one should redo the study with the full form of Eq. (3), but only a parameter study can give a definite answer. Note that adding more detailed measurements to the database will give us the opportunity to compare the details of the theory over a wide range of plasma parameters and size. This is needed if one hopes to distinguish between factors of 2-3 in the theory.

\section{Seed island}

The neoclassical modes need ${ }^{6}$ a seed island of width above the threshold value $w_{\text {crit }}$, as shown in Fig. 3. This is well correlated experimentally with other MHD events such as sawteeth, edge-localized modes (ELMs) and fishbones. Two examples are shown in Fig. 7, from DIII-D, where a sawtooth crash triggers a 3/2 mode, Fig. 7(a), and an ELM provides a seed perturbation for a $2 / 1$ mode in Fig. 7(b). Experimentally, the typical size of the seed island driven by a sawtooth crash or an ELM has not been systematically studied and the scaling with plasma parameters is not well known. It also depends on the difference in toroidal rotation between the $q=1$ and the $q=m / n$ surfaces, for example, for 


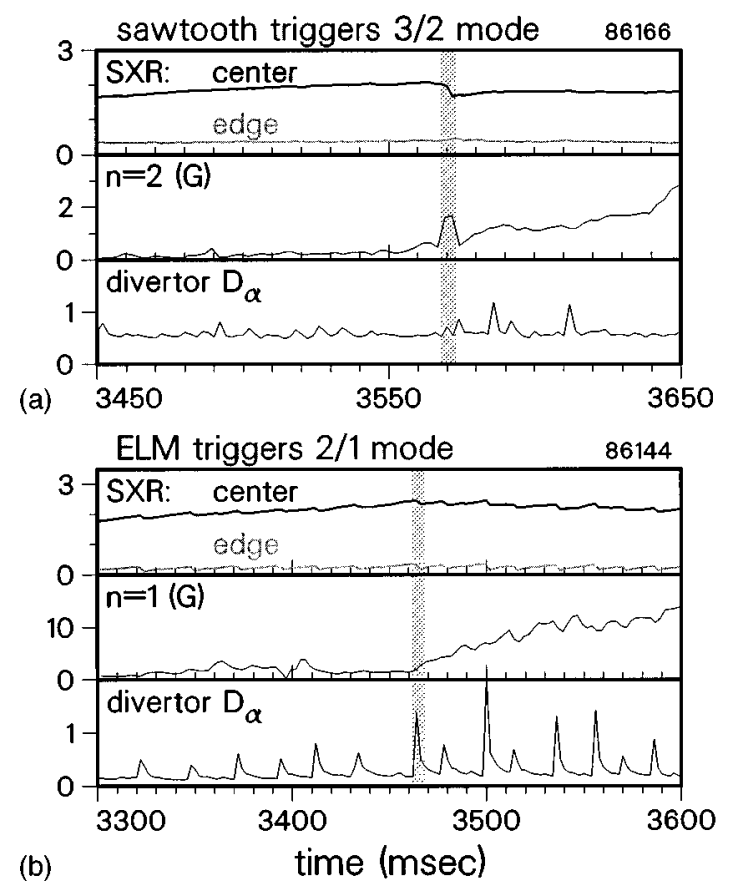

FIG. 7. Correlation of a sawtooth crash (a) with the growth of a 3/2 mode, and of an ELM (b) with the destabilization of a 2/1 mode in DIII-D. This is typical of most tokamaks. In TFTR, the onset of the modes is sometimes related to fishbones activity.

the sawtooth crash, as well as on kinetic effects. Moreover, the theoretical value of $w_{\text {crit }}$ depends on the detailed form of the GGJ term, the polarization term, and of $\chi_{\perp}$ and $\chi_{\|}$in the definition of $w_{d}$, which are still under investigation. If $w_{d \mathrm{GGJ}}=w_{d}$, then the $w_{\text {crit }}$ one obtains using only the " $\chi_{\perp}$ " model, that is neglecting the polarization term, is typically of a few millimeters, as can be seen in the example in Fig. 3(a), which is too small. This shows that all the terms in Eq. (3) have to be revised for small $w$. Note also that the perturbation at the $q=m / n$ due to a sawtooth or an ELM vary when varying the plasma parameters, in particular as the reconnection time varies. Therefore, while in qualitative agreement with the theory, a quantitative analysis of the onset of the neoclassical modes, the scaling of the seed island size with plasma parameters, and the physics of the seed island formation are still needed. In particular, we have seen that one typically sees these neoclassical modes at beta values two to three times higher than the critical limit, at which any seed island larger than $w_{\text {marg }}\left(\approx w_{d}\right)$ can be destabilized. Thus, it indicates that the maximum experimentally observed available $w_{\text {seed }}$ is typically smaller than $w_{\text {marg }}$, and its actual size determines the value of beta at the onset of the mode. Therefore $w_{d}\left(\approx w_{\text {marg }}\right)$ is a good measure of an upper bound for the island threshold and would therefore be of the order of 2-5 cm in ITER (up to 6-7 cm with the maximum polarization term taken into account), including the uncertainty in the definition of $w_{d}$.

\section{PROJECTION TO ITER}

In order to study the long-pulse beta limit in ITER DIII-D made a systematic study of ITER-like sawtoothing, ELMy H-mode discharges with $q_{95} \approx 3-4$, and varying the

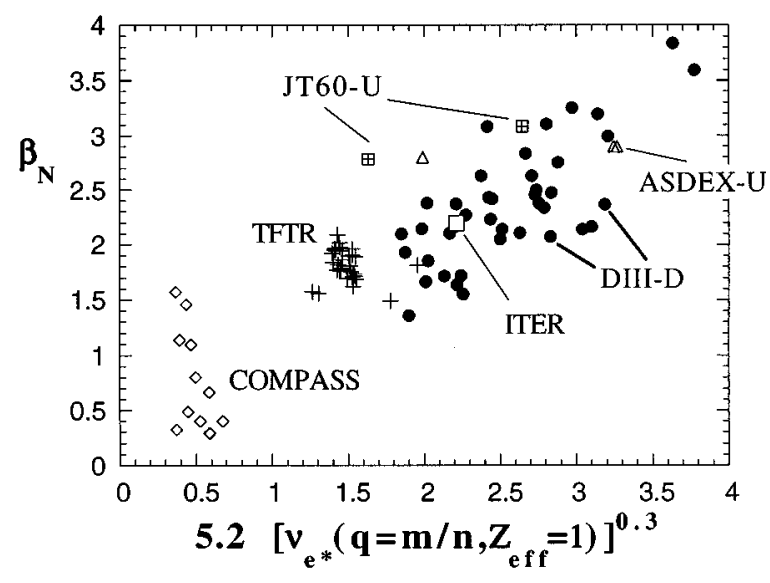

FIG. 8. Here $\beta_{N}$ versus local $\nu_{e} *\left(Z_{\text {eff }}=1\right)$ at the onset of the $q=m / n$ neoclassical tearing mode from the ITERLPBL.DB1 database. Typical values of $m / n$ are $3 / 2,4 / 3,2 / 1$, and $5 / 2$. The ITER point is obtained from the $q=2$ plasma parameters, and with $Z_{\text {eff }}=1$. The scaling was suggested by the DIII-D data in Ref. 7.

density in order to reach ITER collisionality. ${ }^{7}$ The DIII-D experimental scaling of the onset of the $3 / 2$ and, in particular, the $2 / 1$ modes is given by $\beta_{N} \sim \nu_{e^{*}}^{0.32 \pm 0.02} \rho_{*}^{0.13 \pm 0.15}$. Since no unambiguous $\rho_{*}$ dependence can be singled out from the data, we show in Fig. $8 \beta_{N}$ vs $5.2 \nu_{e^{*}}^{0.3}$ at the onset of the $\mathrm{m} / \mathrm{n}$ modes, with $\nu_{e^{*}}\left(Z_{\text {eff }}=1\right)=0.012 n_{e}\left(10^{20} \mathrm{~m}^{-3}\right) q R(\mathrm{~m}) /$ $\epsilon^{3 / 2} T_{e}^{2}(\mathrm{keV})$, where the local data at $q=m / n$ from the database are used. Typical values of $m / n$ are $3 / 2$ and $4 / 3$ for ASDEX-U, TFTR, $3 / 2$ and $2 / 1$ for DIII-D, $2 / 1$ for COMPASS-D, and 5/2 and 3/1 for JT60-U. One should incorporate the effect of $Z_{\mathrm{eff}}$ in $\nu_{e^{*}}$, however, these values are not complete in the database at present. Therefore we used $Z_{\text {eff }}=1$, as was done in Ref. 7. This would, for example, move the JT60-U points to the right as $Z_{\text {eff }}$ is about twice as large as in the other shots. However, one sees a clear dependence on $\nu_{e^{*}}$, even if there is a large scatter of the data, and if each machine might have a different scaling. This is due to the fact that it is very sensitive to the local profiles and moreover to the formation of the seed islands. For example, the JT60-U long-pulse discharges have $q_{\text {min }} \approx 2$, using currentdrive and weak/negative magnetic shear, and no sawtooth activity. The neoclassical tearing modes are typically seen after giant ELMs, which is why JT60-U was able to increase its operational beta limit by increasing the plasma triangularity. ${ }^{4}$ One also sees from Fig. 8 that the COMPASS-D data do not depend on $\nu_{e^{*}}$, but that another mechanism is responsible for determining the onset of the modes. It was shown ${ }^{8}$ that this was consistent with $\nu_{i} / \epsilon \omega_{e^{*}}$ becoming smaller than a constant value of about 0.3 , which is a change in the value of the polarization term. Finally, we also note that for some of the discharges, the ideal MHD limit was computed in order to make sure that these modes were not due to a lower than expected ideal limit due to unfavorable profiles. A limit consistent with $\beta_{N} \approx 4 l_{i}$ was found (well above the actually observed limit) for the external kink and with no wall stabilization.

An ITER reference point is also shown, where values at the $q=2$ surface of an ignited scenario have been used 
$\left(n_{e 20}=1.3, \quad T_{e}=T_{i}=5.1 \mathrm{keV}, \quad \rho_{s}=2.4 \mathrm{~m}, \quad I_{p}=21 \mathrm{MA}\right.$, $R_{0}=8.1 \mathrm{~m}, \quad a=2.8 \mathrm{~m}, \quad n_{e 0,20}=1.3, \quad T_{e 0}=20 \mathrm{keV}$, $T_{i 0}=19 \mathrm{keV}, B_{0}=5.6 \mathrm{~T}, Z_{\mathrm{eff}}=1.5, \beta_{N}=2.2$, and $\left.\beta_{p}=0.7\right)$. One sees that the ITER point lies in the middle of what can be called the "gray region," where neoclassical modes have been observed. Two points should be mentioned about this plot.

(1) Each of the tokamaks have similar discharges that do not have low $-m / n$ resistive modes and have been sustained for many energy confinement times, however, no discharges, near ITER collisionality, have been obtained with a $\beta_{N}$ significantly larger and close to the ideal limit, which would be an approximately horizontal line at 3.5-4 in Fig. 8. Therefore the "gray region" represents the operational domain where neoclassical may or may not occur depending on the local profiles and the available seed islands. ${ }^{3}$

(2) While the points are the values at the onset of the mode and most of the discharges, except for those with the 2/1 modes in DIII-D, can be sustained for many $\tau_{\text {th }}$ with these saturated modes, the sustained beta is in general at the same value or slightly smaller due to the drop in confinement time induced by the saturated island. If the power is increased, $\tau_{\text {th }}$ is further reduced and the actual beta value stays the same.

Note that everything else being constant, one expects the saturated island width to be proportional to $\left(1+\nu_{e^{*}}^{1 / 2}\right)$, due to the collisionality dependence of the bootstrap current coefficients, and therefore the operational or soft beta limit as well. From Fig. 8, one expects the ITER soft beta limit to be in the range $\beta_{N} \approx 2-2.5$. One can change this limit by profile control, as well as by avoiding the perturbations causing the large seed islands, as was done for example in JT60-U, where they have increased $q_{\min }$ above 1 and have no sawteeth, as mentioned above. However, keeping $q_{0}>1$ might require substantial current drive and removing the ELMs would cause problems in particle control in ITER, so that a better operational regime is "a $\mathrm{H}$ mode with frequent and small amplitudes ELMs and sawteeth.' In JET (Joint European Torus), they have been able to sustain long-pulse discharges at $\beta_{N}$ values up to $3 .^{37}$ This might be a challenge to the neoclassical theory and should be studied in detail. However, the local collisionality is somewhat larger than the ITER value and therefore these data points tend to lie within or near the "'gray region." Moreover, as the fraction of fast particles was rather high, one needs to study their effects on the stability of the neoclassical modes, which can be even more important for ITER.

A crude estimation of the perturbation at the $q=m / n$ surface triggered by a sawtooth "gong,",38 from toroidal coupling without rotation effects, and assuming $\delta B_{r, m n} \approx 0.5\left(a / \rho_{s}\right)^{m}\left(\rho_{s} / 2 R\right) \delta B_{\theta, m-1, \text { edge }}$, gives $w_{\text {seed, } m n}$ $=\left[4 \rho_{s}^{2}\left(a / \rho_{s}\right)^{m} \delta B_{\theta, m-1, \text { edge }} /\left(n B_{0} s\right)\right]^{1 / 2}$, with $B_{0}$ the axial toroidal field and $\delta B_{\theta, m \text {,edge }}$ the $\mathrm{m} / \mathrm{m}$ component of the edge perturbed poloidal magnetic field due to the sawtooth crash. Using values at the $q=2$ surface, since the $2 / 1$ mode is the most probable mode to cause a disruption, one obtains $w_{\text {seed }}(\mathrm{ITER}, m / n=2 / 1) \approx 1.7\left[\delta B_{\theta, 1, \text { edge }}(\mathrm{G})\right]^{1 / 2}(\mathrm{~cm})$.

Therefore, as $w_{\text {crit }} \leqslant 6 \mathrm{~cm}$, one can expect neoclassical modes if the perturbation at the edge due to the sawtooth "gong" is of the order of $10 \mathrm{G}$, which is quite realistic. One sees from this crude estimate that a more precise scaling is needed in order to predict ITER performance.

If $w_{\text {seed }}>w_{\text {crit }}$ is satisfied, then one can define the hard beta limit as the beta value at which the saturated island width of a given mode is of the order of its distance to the edge. This criterion was used in Ref. 8 to successfully model the dependence on $q_{95}$ of the hard beta limits in ASDEX-U and COMPASS-D. Following this criteria, $\beta_{N}=2.2$ is an upper bound for ITER as the saturated island width would be $60-70 \mathrm{~cm}$ at the $q=2$ surface, whereas $a-\rho_{s}=60 \mathrm{~cm}$. Using Fig. 5(b), $\tau_{\mathrm{R}}=800 \mathrm{~s}, w_{\text {sat }} / \rho_{s}=25 \%$, and $\left|\rho_{s} \Delta^{\prime}\right|=2$, one sees that the island would grow as follows:

It would reach $30 \mathrm{~cm}\left(w / w_{\text {sat }}=0.5\right)$

$$
\text { in } t=0.08 * 800 / 2 \approx 30 \mathrm{~s} \text {. }
$$

It would reach $55 \mathrm{~cm}\left(w / w_{\text {sat }}=0.9\right)$

$$
\text { in } t=0.40 * 800 / 2 \approx 150 \mathrm{~s} \text {. }
$$

Therefore, while being a hard beta limit, there is sufficient time to detect the mode and stop the discharge before the disruption or try to stabilize or reduce the saturated width with a feedback system. A possible feedback mechanism is to replace the reduction of the bootstrap current inside the island by electron cyclotron current drive (ECCD), as this system can provide highly localized current deposition. This can stabilize the mode completely. The typical bootstrap current density in ITER reference scenarios is $j_{\mathrm{bs}} \approx 0.1 \mathrm{MA} / \mathrm{m}^{2}$, thus one has the following current to replace, depending on the island size $w_{\text {sat }}$ :

$$
I_{\mathrm{bs}}\left(q=2, w_{\mathrm{sat}}\right) \approx 2 \pi \rho_{s} \kappa w_{\mathrm{sat}} j_{\mathrm{bs}} \approx 5\left(w_{\mathrm{sat}} / \rho_{s}\right) \quad(\mathrm{MA}) .
$$

On the other hand, a value of $w_{\text {sat }} / \rho_{s}$ of $10 \%$ gives a $20 \%$ drop in confinement time, which is the maximum value acceptable to maintain a reasonable performance. Therefore one has to provide $500 \mathrm{kA}$ of ECCD in a normalized width of $10 \%$. The driven current is given by the efficiency $\gamma$ $\left(10^{20} \mathrm{~m}^{-2} \mathrm{MA} / \mathrm{MW}\right)$ and one has the following requirements:

$$
I_{\mathrm{ECCD}}=\frac{\gamma P(\mathrm{MW})}{n_{e}\left(10^{20} \mathrm{~m}^{-3}\right) R(\mathrm{~m})}=I_{\mathrm{bs}}=5 \frac{w_{\mathrm{sat}}}{\rho_{s}} \approx 5 \frac{\Delta \rho}{\rho},
$$

where $\Delta \rho / \rho$ determines the relative localization of the current profile. Therefore, assuming that $50 \mathrm{MW}$ are available, $n_{e 20}=1$ and $R=8$, an effective current efficiency,

$$
\gamma_{\mathrm{eff}} \equiv \frac{\gamma}{\Delta \rho / \rho} \approx 0.8
$$

is required. This $\gamma_{\text {eff }}$ versus the normalized radius where the current profile peaks, $\rho_{\text {peak }} / a$, is shown in Fig. 9(a) for a launch $2 \mathrm{~m}$ above the equatorial plane and a toroidal angle varied from 0 (perpendicular) to $45^{\circ}$ along each curve. The poloidal angle with respect to the vertical line is a parameter and is varied from $80^{\circ}$ to $130^{\circ}$, as indicated. One sees that 

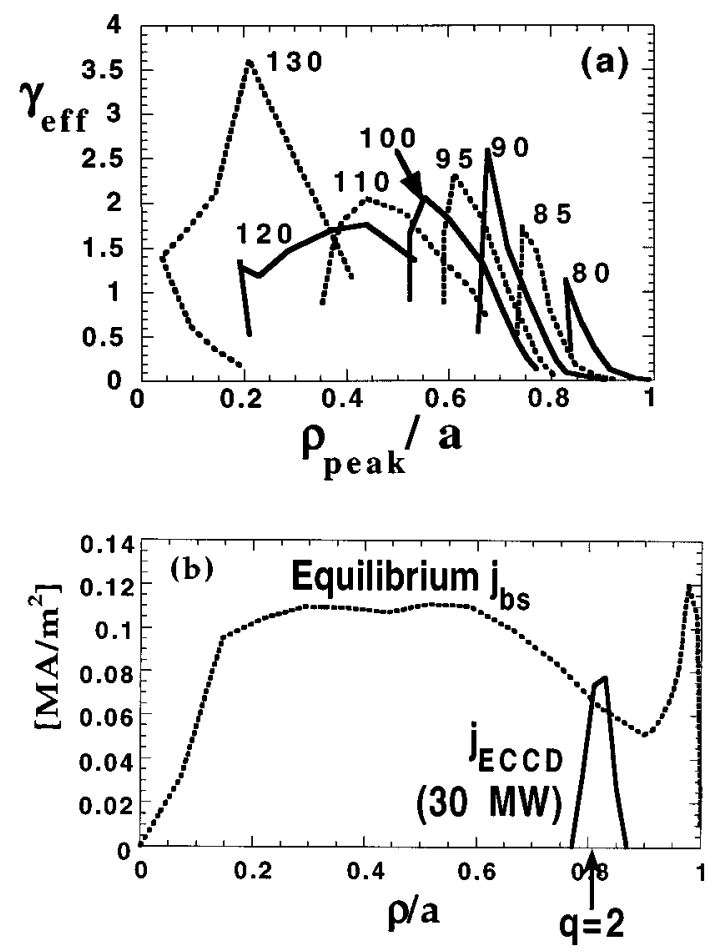

FIG. 9. (a) Effective current-drive efficiency, $\gamma_{\text {eff }}$, as computed with the code TORAY normalized to peakedness of the deposition profile, versus the position of the profile peak, using an ITER equilibrium. The parameter is the poloidal angle, and the curves are obtained by varying the toroidal launch angle. (b) Corresponding ITER equilibrium bootstrap current density and ECCD density obtained with $P=30 \mathrm{MW}$, versus normalized radius, for $\theta_{\mathrm{pol}}=80^{\circ}$ and $\varphi_{\mathrm{tor}}=15^{\circ}$.

$\gamma_{\text {eff }}>0.8$ can be obtained at $\rho_{s}(q=2) / a=0.8$. Note that this is a preliminary study using the present design specifications for the ITER ECRH system. The efficiency can be optimized as well as the actual requirement for $\gamma_{\text {eff }}$ reduced when the exact geometry of the island and the possibility of modulating the rf power in phase with the island $\mathrm{O}$ point are taken into account. Also shown in Fig. 9(b) is the equilibrium bootstrap current and the ECCD driven with $30 \mathrm{MW}$ of ECRH power. This shows that it is possible to substitute the bootstrap current near the $q=2$ surface, with $\Delta \rho / \rho \leqslant 0.1$, within the ITER design parameters. Also, Fig. 9(a) shows that other modes, with lower $q=m / n$ values, can be stabilized. However, the feasibility of such a feedback mechanism has to be demonstrated in present experiments first. Such experiments are underway in COMPASS-D and will be studied in ASDEX-U and DIII-D. The possible local modification of the current gradient by either ECCD and/or fast wave current drive could also be used to reduce $\Delta^{\prime}$, i.e., make it more negative, thus raising the neoclassical destabilization threshold, or to change the sawtooth behavior.

\section{CONCLUSIONS}

An extensive study of the conventional and neoclassical resistive theories has been presented, including an integration of all the previously published terms into a single island evolution equation. Most of the terms are valid for noncircular toroidal MHD equilibria and a comparison with local data from ASDEX-U, COMPASS-D, DIII-D, JT60-U, and TFTR has also been shown. We have confirmed that the driving term, namely the perturbed bootstrap current due to the flattening of the pressure profile in the island, is well defined theoretically for large islands and predicts well the experimental saturated island widths. We have also pointed out the present limitations of the theory in order to predict the island threshold width, but have proposed an upper limit corresponding to the width at the maximum driving term.

Several tokamaks are limited in their long-pulse discharges to beta values much lower than the ideal limit due to low- $m / n$ resistive modes. These modes have been identified as the neoclassical tearing modes. We have shown that the hysteresis behavior of these modes between $\beta_{p \text {, onset }}$ and $\beta_{p \text {,crit }}$ is a characteristic that helps to distinguish them from the conventional resistive tearing modes. We have also seen that the error in the $\Delta^{\prime}$ calculations need to be smaller than $\pm m / \rho_{s}$ in order to be able to distinguish between the conventional and neoclassical growth of the island. However, the decay and stabilization of the mode at $\beta_{p} \approx \beta_{p \text {,crit }}$ clearly identifies the mode as being driven by the perturbed bootstrap current, since the $\beta$ dependence of $\Delta^{\prime}$ is too weak to explain the island evolution, and the GGJ term cannot explain the evolution either.

These modes need a seed island of size $w_{\text {seed }}>w_{\text {crit }}$. The growth of the neoclassical modes are well correlated with other MHD events and are often triggered by sawtooth crash, ELMs, or fishbones. The exact form of $w_{\text {crit }}$ needs further theoretical work, and the scaling of $w_{\text {seed }}$ with plasma parameters has to be quantified experimentally. However, in comparing with results from ASDEX-U, COMPASS-D, DIII-D, JT60-U, and TFTR, we have shown that $w_{d}$, which measures the location where the drive is maximized, is a good estimate of the upper bound for $w_{\text {crit }}$. The lower limit of $w_{\text {crit }}$ is not well determined but is of the order of $\epsilon^{1 / 2} \rho_{p}$. This gives a maximum value of $2-6 \mathrm{~cm}$ for ITER, that is $w_{d} / \rho_{s} \approx 0.01-0.02$. Therefore sawtooth and ELM control capabilities should be investigated for ITER, in order to be able to avoid these modes.

If the modes cannot be avoided, a limit of $\beta_{N}=2-2.2\left(\beta_{p} \approx 0.7\right)$ is predicted for ITER since, at this beta value, the saturated island size at the $q=2$ surface is of the order of the distance to the edge and will cause a disruption. However, due to the very large resistive time in ITER, the island will need 150-200 s to grow to its full size. Therefore one has operational time to detect the mode and end the discharge before the disruption, since one needs less than 30 $\mathrm{s}$ to decrease $\beta$ by a factor $10,{ }^{39}$ or control the final saturated size with an external feedback mechanism. Such a system could be ECCD, in order to replace the reduction of the bootstrap current inside the island. We have shown that this is possible with $50 \mathrm{MW}$ of ECRH power, using the present design specifications for the gyrotrons. However, this needs to be first demonstrated in the present experiments. Other possible control mechanisms are keeping $q_{0}>1$, reverse shear configurations, since the neoclassical tearing modes are stable for $q^{\prime}<0$, and profile control since they are very sensitive to local parameters.

Further studies are underway to better quantify the pre- 
dictions to ITER, in particular, concerning the experimental scaling of the typical seed islands due to sawteeth and ELMs, and the theoretical prediction of $w_{\text {crit }}$, including kinetic and rotation effects. Nonlinear three-dimensional (3D) MHD simulation of the island growth in the present experiments are also in progress to determine if nonlinear effects can limit the final saturated island size. Finally, the reproducibility of the discharges with and without modes in the "gray region" will indicate the level of plasma and profile control needed in ITER to avoid the modes in long-pulse discharges and to sustain the adequate target scenario: a long-pulse $\mathrm{H}$ mode with small and frequent sawteeth and ELMs.

\section{ACKNOWLEDGMENTS}

The ASDEX-U, COMPASS-D, DIII-D, JT60-U, and TFTR teams are acknowledged for providing very useful data to the ITER long-pulse beta limit database. One of the authors (OS) is grateful to D. Campbell and C. Challis for discussions about the JET results, and to the referee for useful comments.

The work by OS was funded in part under the VHTP task Agreement No. S 19 TD 0195-07-24FE and by the Swiss National Science Foundation. The work by RWH was supported under U.S. Department of Energy Contract No. DE-AC03-94SF20282 and under Raytheon Engineers and Constructors, Inc. Subcontract No. ITER-GA-4002.

${ }^{1}$ E. J. Strait, Phys. Plasmas 1, 1415 (1994).

${ }^{2}$ E. J. Strait, T. S. Taylor, A. D. Turnbull, J. R. Ferron, L. L. Lao, B. Rice, O. Sauter, S. J. Thompson, and D. Wroblewski, Phys. Rev. Lett. 74, 2483 (1995).

${ }^{3}$ Z. Chang, E. D. Fredrickson, J. D. Callen, K. M. McGuire, M. G. Bell, R. V. Budny, C. E. Bush, D. S. Darrow, A. C. Janos, L. C. Johnson, H. K. Park, S. D. Scott, J. D. Strachan, E. J. Synakowski, G. Taylor, R. M. Wieland, M. C. Zarnstorff, S. J. Zweben, and the TFTR Team, Nucl. Fusion 34, 1309 (1994).

${ }^{4}$ Y. Kamada, K. Ushigusa, Y. Neyatani, O. Naito, T. Ozeki, Y. Kawano, R. Yoshino, H. Kubo, T. Fujita, S. Ishida, M. Sato, Y. Koide, S. Takeji, S. Sakurai, K. Tsuchiya, J. Manickam, M. Matsuoka, M. Kikuchi, M. Mori, H. Ninomiya, M. Azumi, and HT-60 Team, in Ref. 10, Vol. 1, p. 651; and "High triangularity discharges with improved stability and confinement in JT-60U," to appear in Ref. 7, Paper No. IAEA-CN-64/A1-6.

${ }^{5}$ H. Zohm, M. Alexander, R. Buttery, S. de Peña Hempel, O. Gruber, T. C. Hender, T. Kass, M. Maraschek, W. Sandmann, S. Sesnic, M. Sokoll, A. Stäbler, H. R. Wilson, H. P. Zehrfeld, ASDEX Upgrade-Team, and NBITeam, " $\beta$-limiting phenomena in ASDEX Upgrade," to appear in Proceedings of the 23rd EPS Conference on Controlled Fusion and Plasma Physics, Kiev, Ukraine, 1996 (European Physical Society, Petit-Lancy, in press).

${ }^{6}$ Z. Chang, J. D. Callen, E. D. Fredrickson, R. V. Budny, C. C. Hegna, K. M. McGuire, M. C. Zarnstorff, and TFTR Group, Phys. Rev. Lett. 74, 4663 (1995).

${ }^{7}$ R. J. La Haye, J. D. Callen, M. S. Chu, S. Deshpande, T. A. Gianakon, C. C. Hegna, S. Jardin, L. L. Lao, J. Manickam, D. A. Monticello, A. Pletzer, A. H. Reiman, O. Sauter, E. J. Strait, T. S. Taylor, A. D. Turnbull, and H. R. Wilson, "Practical beta limit In ITER-shaped discharges in DIII-D and its increase by higher collisionality," to appear in Proceedings of the 16th International Conference on Plasma Physics and Controlled Nuclear Fusion, Montreal, Canada, 1996 (International Atomic Energy Agency, Vienna, in press), Paper No. IAEA-CN-64/AP1-21.

${ }^{8}$ D. A. Gates, B. Lloyd, A. W. Morris, G. McArdle, M. O’Brien, M. Valovic, C. D. Warrick, H. R. Wilson, and the COMPASS-D and ECRH Teams, " $\beta$-limit studies and the effect of error fields at low collisionality on COMPASS-D," to appear in Ref. 7, Paper No. IAEA-CN-64/AP1-17; and H. R. Wilson, M. Alexander, J. W. Connor, A. M. Edwards, D. Gates, O. Gruber, R. J. Hastie, C. C. Hegna, T. C. Hender, R. J. La Haye, L. L. Lao, A. W. Morris, C. M. Roach, E. J. Strait, T. S. Taylor, M.
Valovic, and H. Zohm, Plasma Phys. Controlled Fusion 38, A149 (1996). ${ }^{9}$ Z. Chang and J. D. Callen, Nucl. Fusion 2, 219 (1990).

${ }^{10}$ M. Rosenbluth, J. Hogan, D. Boucher, P. Barabaschi, A. Bondeson, B. Coppi, L. Degtyarev, S. Haney, H. Goedbloed, T. C. Hender, H. Holties, G. Huysmanns, W. Kerner, J. Manickam, A. Martynov, S. Medvedev, D. Monticello, T. Ozeki, L. D. Pearlstein, F. Perkins, A. Pletzer, F. Porcelli, P.-H. Rebut, S. Tokuda, A. Turnbull, L. Villard, J. Wesley and ITER JCT and Home Teams, in Plasma Physics and Controlled Nuclear Fusion Research 1994, Proceedings of the 15th IAEA Conference, Seville, 1994 (International Atomic Energy Agency, Vienna, 1995), Vol. 2, p. 517.

${ }^{11}$ See National Technical Information Service Document No. DE6008946 (W. Q. Xu and J. D. Callen, University of Wisconsin Plasma Report No. UWPR 85-5, 1985). Copies may be ordered from the National Technical Information Service, Springfield, VA 22161.

${ }^{12}$ R. Carrera, R. D. Hazeltine, and M. Kotschenreuther, Phys. Fluids 29, 899 (1986).

${ }^{13}$ J. D. Callen, W. X. Qu, K. D. Siebert, B. A. Carreras, K. C. Shaing, and D. A. Spong, Plasma Physics and Controlled Nuclear Fusion Research, Kyoto (International Atomic Energy Agency, Vienna, 1987), Vol. 2, p. 157; C. C. Hegna, J. D. Callen, T. A. Gianakon, W. X. Qu, A. I. Smolyakov, and J. P. Wang, Plasma Phys. Controlled Fusion 35, 987 (1993).

${ }^{14}$ R. Fitzpatrick, Phys. Plasmas 2, 825 (1995).

${ }^{15}$ N. N. Gorelenkov, R. V. Budny, Z. Chang, M. V. Gorelenkova, and L. E. Zakharov, Phys. Plasmas 3, 3379 (1996).

${ }^{16}$ A. I. Smolyakov, Sov. J. Plasma Phys. 15, 667 (1989).

${ }^{17}$ M. Zabiégo and X. Garbet, Phys. Plasmas 1, 1890 (1994).

${ }^{18}$ H. R. Wilson, J. W. Connor, R. J. Hastie, and C. C. Hegna, Phys. Plasmas 3, 248 (1996).

${ }^{19}$ F. L. Waelbroeck and R. Fitzpatrick, Phys. Rev. Lett. 78, 1703 (1993).

${ }^{20}$ J. R. Ferron, L. L. Lao, T. H. Osborne, O. Sauter, E. J. Strait, T. S. Taylor, S. J. Thompson, and A. D. Turnbull, in Proceedings of the 21st EPS Conference, Montpellier, 1994 (European Physical Society, Petit-Lancy, 1994), Vol. I p. 86.

${ }^{21} \mathrm{P}$. R. Thomas and the JET Team, "Performance limitations in JET hot-ion H-modes," to appear in Ref. 7, Paper No. IAEA-CN-64/A3-2.

${ }^{22}$ P. H. Rutherford, Phys. Fluids 16, 1903 (1973).

${ }^{23}$ R. B. White and D. A. Monticello, M. N. Rosenbluth, and B. V. Waddell, Phys. Fluids 20, 800 (1977).

${ }^{24}$ A. H. Glasser, J. M. Greene, and J. L. Johnson, Phys. Fluids 18, 875 (1975).

${ }^{25}$ M. Kotschenreuther, R. D. Hazeltine, and P. J. Morrison, Phys. Fluids 28, 294 (1985).

${ }^{26}$ C. M. Bishop, J. W. Connor, R. J. Hastie, and S. C. Cowley, Plasma Phys. Controlled Fusion 33, 389 (1991).

${ }^{27}$ See National Technical Information Service Document No. DE96009530LP (T. A. Gianakon, Computational Modeling of Neoclassical and Resistive MHD Tearing Modes in Tokamaks, PhD thesis, 1996, Univ. Wisconsin Report UW-CPTC 96-1, 1996). Copies may be ordered from the National Technical Information Service, Springfield, VA 22161; T. A. Gianakon, C. C. Hegna, and J. D. Callen, Phys. Plasmas 3, 4637 (1996).

${ }^{28}$ L. A. Charlton, J. A. Holmes, H. R. Hicks, V. E. Lynch, and B. A. Carreras, J. Comput. Phys. 63, 107 (1986).

${ }^{29}$ A. Pletzer, A. Bondeson, and R. L. Dewar, J. Comput. Phys. 115, 530 (1994).

${ }^{30}$ H. Lütjens, A. Bondeson, and O. Sauter, Comput. Phys. Commun. 97, 219 (1996).

${ }^{31}$ C. C. Hegna and J. D. Callen, Phys. Plasmas 1, 2308 (1994).

${ }^{32}$ ITER Confinement Database and Modeling Group, in Ref. 10, Vol. 2, p. 525.

${ }^{33}$ S. P. Hirshmann, Phys. Fluids 31, 3150 (1988).

${ }^{34}$ O. Sauter, Y. R. Lin-Liu, F. L. Hinton, and J. Vaclavik, in Theory of Fusion Plasmas, Proceedings of the Joint Varenna-Lausanne International Workshop, Varenna, 1994, (Editrice Compositori, Bologna, 1994), p. 337.

${ }^{35}$ A. Pletzer, Proceedings Joint Varenna-Lausanne International Workshop, Varenna, 1996 (Editrice Compositori, Bologna, 1997), p. 259.

${ }^{36}$ M. F. Zabiego and J. D. Callen, "Threshold condition for nonlinear tearing modes in tokamaks," to appear in Nucl. Fusion. 
${ }^{37}$ C. D. Challis, B. Balet, V. P. Bhatnagar, J. C. M. de Haas, L.-G. I. Eriksson, M. Gadeberg, C. Gormezano, C. W. Gowers, J. A. How, G. T. A. Huysmans, S. Ishida, W. O. K. Kerner, M. J. Loughlin, F. G. Rimini, G. J. Sadler, A. C. C. Sips, A. Tanga, B. J. D. Tubbing, and D. J. Ward, in Proceedings of the 22nd EPS Conference, Bournemouth, 1995 (European Physical Society, Petit-Lancy, 1995), Vol. II, p. 69.
${ }^{38}$ P. A. Duperrex, A. Pochelon, A. W. Edwards, and J. A. Snipes, Nucl. Fusion 32, 1161 (1992).

${ }^{39}$ D. Boucher, Y. Barano, B. Fischer, X. Litaudon, D. Moreau, W. Nevins, F. Söldner, I. Voitsekhovitch, and the ITER JCT and Home Teams, "ITER scenarios including non-inductive steady-state operation," to appear in Ref. 7, Paper No. IAEA-CN-64/FP-22. 